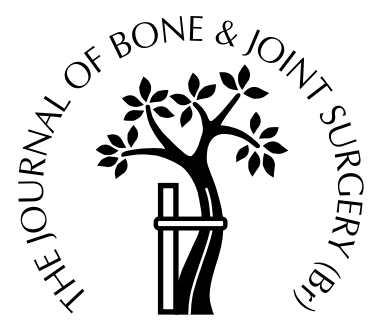

\title{
Cytological diagnosis of skeletal lesions
}

\author{
FINE-NEEDLE ASPIRATION BIOPSY IN 110 TUMOURS \\ Rikard Wedin, Henrik C. F. Bauer, Lambert Skoog, Veli Söderlund, \\ Edneia Tani \\ From the Karolinska Hospital, Stockholm, Sweden
}

W e have previously shown that cytological diagnosis based on fine-needle aspiration biopsy

(FNAB) is a safe and efficient method for the discrimination between benign, primary malignant and metastatic bony lesions. We have now studied metastatic lesions to assess the diagnostic accuracy and to ascertain whether FNAB allows identification of the primary lesion.

Between 1990 and 1997, 447 patients were referred for diagnosis of skeletal lesions of unknown type. Of these 119 proved to have metastatic disease, either myeloma or lymphoma. Nine were excluded leaving 110 consecutive patients with metastatic carcinoma (80), myeloma (16) or lymphoma (14).

FNAB gave a correct diagnosis in 102 of the 110 patients $(93 \%)$. In eight it was inconclusive. It correctly diagnosed 15 of 16 patients with myeloma, 12 of 14 with lymphoma, and 75 of 80 with metastatic carcinoma. Furthermore, the site and type of malignancy were correctly suggested in two-thirds of patients with metastatic carcinoma. Overall, only seven open biopsies were carried out.

We conclude that time-consuming and costly investigations can be reduced by choosing FNAB as the initial diagnostic method for skeletal lesions of unknown origin. The choice of radiological examinations, laboratory tests and surgical biopsies can be determined by using FNAB.

J Bone Joint Surg [Br] 2000;82-B:673-8. Received 19 March 1999; Accepted 18 August 1999

R. Wedin, MD, $\mathrm{PhD}$, Consultant

H. C. F. Bauer, MD, Professor

Orthopaedic Oncology Service, Department of Orthopaedics

V. Söderlund, MD, Consultant

Department of Radiology

L. Skoog, MD, Associate Professor

E. Tani, MD, Associate Professor

Department of Pathology and Cytology, Division of Clinical Cytology

Karolinska Hospital, S-171 76 Stockholm, Sweden.

Correspondence should be sent to Dr R. Wedin.

(C)2000 British Editorial Society of Bone and Joint Surgery 0301-620X/00/59992\$2.00

VOL. 82-B, No. 5, JULY 2000
Bony collapse in a patient without a history of malignant disease is a diagnostic challenge. Differential diagnoses include reactive or inflammatory lesions, sarcoma, metastatic carcinoma, myeloma or lymphoma, but the radiological features are seldom specific enough to allow a conclusive diagnosis to be made. This should be confirmed by histology using either fine-needle aspiration biopsy (FNAB) or open surgical biopsy. In metastatic disease the identity of the primary tumour is important since specific treatment may be available, for example, in metastases from breast, prostate, thyroid and some neuroendocrine carcinomas. In the case of a lesion which suggests metastatic disease, some authors recommend that radiology should be used to identify the primary tumour and that biopsy should only be carried out if this fails to give an answer. $^{1-3}$ This recommendation is based on two assumptions; first, that the tissue obtained from the metastasis seldom provides information about the site of the primary tumour and secondly, that surgical biopsy is associated with significant morbidity.

So far, the use of FNAB for cytological diagnosis of bone lesions has been limited. In a study of 300 patients, we reported correct discrimination between benign, primary malignant and secondary malignant bone lesions in $95 \%$ of adequate fine-needle aspirates. ${ }^{4}$ Similar figures were found by Bommer, Ramzy and Mody ${ }^{5}$ in a study of 427 patients with skeletal lesions. Ruhs, El-Khoury and Chrischilles ${ }^{6}$ found FNAB to be more cost-effective than open biopsy.

The use of FNAB as the initial investigation will allow most benign and primary malignant lesions to be diagnosed. If the cytology is diagnostic of a secondary malignancy the clinician may be guided to the primary tumour. In this report we have therefore focused on skeletal lesions which, after investigation, proved to be metastases, myeloma or lymphoma in an attempt to assess the extent to which FNAB provides a diagnosis and information about the site of the primary tumour.

\section{Patients and Methods}

Between 1990 and 1997, 447 patients were referred to the Orthopaedic Oncology Service of the Karolinska Hospital for the diagnosis of skeletal lesions of unknown type. Of 
these, 146 were diagnosed as having a primary malignant bone tumour, 182 a benign or reactive lesion, and 119 a metastatic carcinoma, myeloma or lymphoma. We selected the last 119 consecutive patients for this study. Nine of these were excluded; three had had a large core needle biopsy before referral, and in six the medical records could not be retrieved. This left 110 patients in the study. Seven had had inconclusive FNAB at other hospitals before referral. There were 68 men and 42 women with a median age of 67 years ( 4 to 87 ). The sites of the tumour were the pelvis (39), long bones (36), spine (20) and others (15).

FNAB was carried out as an outpatient procedure at the first visit. Except for tumours with an extraskeletal palpable mass, this was usually done under radiological guidance by a cytologist in collaboration with a radiologist. Occasionally, CT-guided aspiration was used for vertebral lesions. A $0.5 \mathrm{~mm}$ needle $(25 \mathrm{G})$ with a stylet was employed in most cases. Each aspirate was smeared on to slides, air-dried and stained with May-Grünewald-Giemsa (MGG). The yield was immediately checked macroscopically and in faststained MGG smears. If necessary, the aspiration was repeated. In 45 cases (25 metastatic carcinoma, 11 myeloma and 9 lymphoma) the material was suspended in buffered saline and used for preparation of cytospin slides for immunocytochemistry. One patient developed erysipelas the day after FNAB of the femur. There were no other complications. The cytological diagnosis was compared with a consensus diagnosis based on radiological and clinical findings and histological postoperative specimens, as well as information from the Karolinska Hospital. There were 80 patients with metastatic carcinoma, 14 with lymphoma, and 16 with myeloma. In 69 of the 80 patients with metastatic carcinoma, the primary tumour was identified in the lung (26), kidney (26), prostate (6), breast (2), and other sites (9). The primary site of malignancy was not found in 11 patients.

The medical records of all patients were reviewed to assess the time between the onset of symptoms and the first consultation, and the time between first consultation and referral to our department. Assessments of radiographs by radiologists in our hospital were recorded in 95 cases. We recorded all investigations to identify the primary tumour before FNAB. We did not include radiological studies directed towards the specific skeletal lesion or chest radiographs, bone scans or common laboratory tests since we consider these to be standard procedures for all patients. No patient was lost to follow-up. Survival after diagnosis was assessed using the Kaplan-Meier method. Dates of death were obtained from the regional tax authorities.

\section{Results}

Patient history. The median delay between the onset of symptoms and the first medical consultation was four weeks and after another median delay of six weeks (0 to $61)$ the patients were referred to our service. A total of 60 patients was referred without specific prior investigations. In the remaining 50 patients, 94 investigations had been carried out to find the primary tumour. These included abdominal ultrasound (23), endoscopy (11), mammography (11), electrophoresis (11), abdominal CT (10), radiographs of other parts of the skeleton (13), gynaecological examination (6), other radiological investigations (6) and biopsies of other sites (3). These investigations did not contribute to the diagnosis of metastasis or to identification of the primary tumour.

A correct cytological diagnosis with subtyping of the tumour was given in 102 of the 110 patients (93\%). In one, the initial cytological diagnosis and the consensus diagnosis were identical while the primary histological diagnosis was misleading. Of the 102 patients with a correct diagnosis by FNAB, the origin of the primary tumour was established in 79 , but in 23 no suggestion as to the primary site was made. There was no particular site from which it proved too difficult to obtain conclusive material for cytological diagnosis. Overall, only seven surgical biopsies had been undertaken in the 110 patients.

FNAB was inconclusive in five patients, misleading in one and disagreed with the histopathological diagnosis in two (Table I). Of the inconclusive cases, three had nonrepresentative material and in two the FNAB aspirate showed only necrotic tumour cells which could not be classified further. In all eight patients the final diagnosis was made on histological material from surgical specimens and the diagnostic difficulties had no major clinical consequences.

In 75 of 80 patients with metastatic carcinoma, the FNAB diagnosis was conclusive. In 48 of the 80 patients cytomorphology indicated the site of the primary malignancy. The primary tumour was correctly identified in 45 . In two patients, the suggested site for the primary tumour could not be verified and in one, a different carcinoma was found. FNAB correctly identified the origin of the metastasis in 22 of the 26 patients who were found to have renal carcinoma, in 13 of the 26 patients with lung cancer and in five of six patients with prostatic carcinoma. Renal carcinoma will, in most cases, present with a typical cytomorphological picture, while other adenocarcinomas are more difficult to subclassify (Fig. 1). Two patients were diagnosed as having a sarcoma.

FNAB correctly diagnosed the tumour in 15 of the 16 patients with myeloma and in 12 of the 14 with lymphoma. All cases of lymphoma were high grade, and characterised by blastic cells of $\mathrm{B}$ phenotype with light chain restriction (Figs 2 to 4). Only necrotic material was present in aspirates from one patient with myeloma in a vertebra and another with pelvic lymphoma. The third case was diagnosed as most likely to be a metastatic carcinoma.

Radiological assessment. The radiological assessments of 95 patients were recorded. Half of the cases of collapse were diagnosed as metastases and the other half as nonspecific malignancy or collapse. One was diagnosed as 


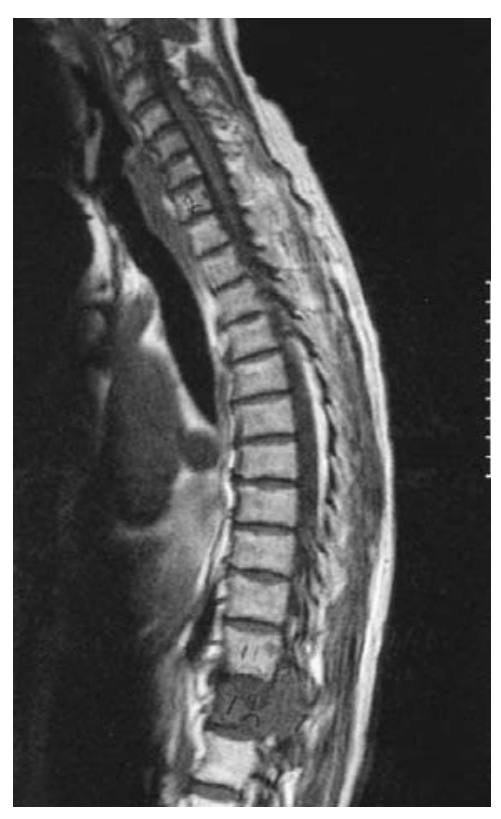

Fig. 1a

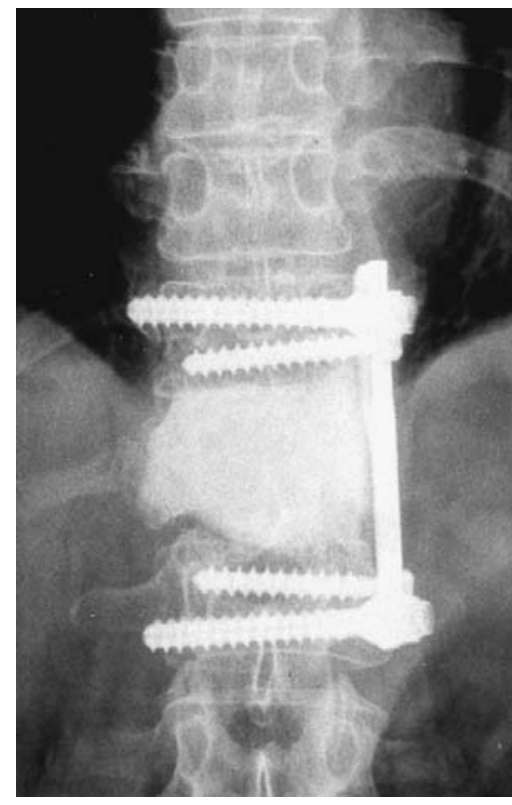

Fig. 1b

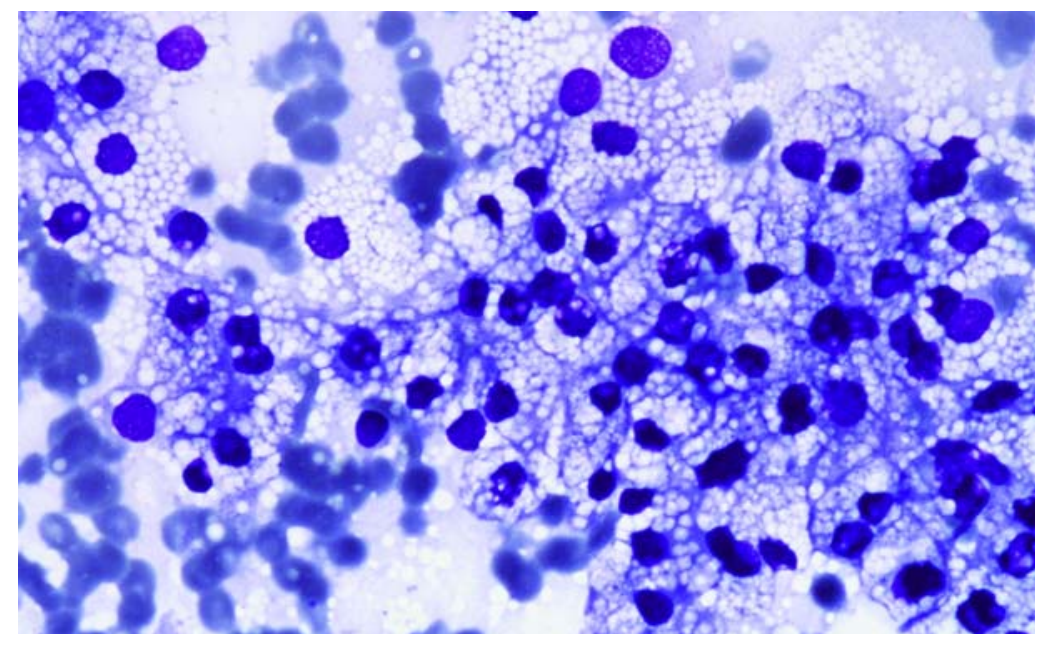

Fig. 1c
Figure 1a - A 72-year-old woman had pain in the right hip and back for almost a year before diagnosis. The initial spinal radiographs were negative and she was treated by physiotherapy and nerve blocks because of the intense pain. Mammography and gynaecological examination were also performed. Finally, a bone scan showed a lesion in the twelfth thoracic vertebra. Radiographs and MRI confirmed a suspected solitary metastasis. Figure $1 \mathrm{~b}-$ The patient had severe pain, but no neurological deficits. No other metastases were detected and she was treated surgically by nephrectomy and removal of the twelfth thoracic vertebrae through an anterior approach. Reconstruction was by cement and a plate. She had a further procedure 2.5 years later because of recurrent compression of the cord and died four years after the diagnosis. Figure $1 \mathrm{c}-$ FNAB smear showing metastatic renal carcinoma characterised by clusters of clear cells with a vacuolated large cytoplasm and rounded central nucleus $(\mathrm{MGG} \times 80)$.

Table I. Analysis of cases which were inconclusive or in disagreement

\begin{tabular}{llll} 
& & Diagnosis & \\
\cline { 3 - 4 } Site & FNA & Histological & Consensus \\
\hline Femur & Non-representative material & Renal carcinoma & Renal carcinoma \\
Pelvis & Non-representative material & Prostate carcinoma & Prostate carcinoma \\
Pelvis & Non-representative material & Lymphoma & Lymphoma \\
Vertebrae & Necrotic tumour cells & Myeloma & Myeloma \\
Femur & Necrotic tumour cells & Renal carcinoma & Renal carcinoma \\
Femur & Sarcoma & Synovial sarcoma & Paraganglioma of the pancreas diagnosed one year \\
& & & later \\
Femur & Carcinoma or sarcoma & Carcinoma or sarcoma & Renal carcinoma diagnosed three years later \\
Femur & Carcinoma & Lymphoma & Lymphoma \\
\hline
\end{tabular}

benign. The diagnosis of a primary tumour based on the radiological appearance of the skeletal metastases was seldom successful.

Operative treatment. Of the 110 patients, 33 had operations for skeletal lesions. Of these, 12 had a pathological fracture, 15 an impending fracture and the remaining six were operated on for epidural spinal metastases. Six patients had lung carcinoma, 12 renal carcinoma, five myeloma and nine metastatic carcinoma from other sites. Only one patient with lymphoma had surgery. The most common site treated by operation was the femur (11) followed by the humerus (8), spine (6), pelvis (4) and other 
sites (4). Five en-bloc resections for solitary metastases of the femur or humerus were carried out, all in patients with renal carcinoma and long life expectancy.

Clinical outcome. Of the 110 patients, 28 are still alive with a median follow-up of two years (1 to 8). The median survival time from diagnosis for the whole series of 110 patients was 1.5 years. Patients with myeloma and lymphoma had the highest rates of survival, with chance of being alive at one year of 0.9 (95\% CI 0.88 to 1$)$ and $0.6(95 \%$ CI 0.46 to 0.74 ), respectively (Fig. 5). Patients with metastatic carcinoma had a considerably worse prognosis, with a oneyear rate of survival of 0.42 (95\% CI 0.36 to 0.48$)$. Of patients with lung carcinoma $50 \%$ had died within three months of diagnosis while the rate of survival for patients with renal carcinoma was 0.6 (95\% CI 0.50 to 0.70$)$ at one year.

\section{Discussion}

In most instances, skeletal metastasis, myeloma and lymphoma can be reliably diagnosed by FNAB. It is a simple outpatient procedure which offers sufficient material for diagnosis in more than nine of ten patients. Moreover, our study shows that FNAB gives guidance in the search for the primary lesions; 27 out of 30 myelomas or lymphomas were diagnosed by FNAB and in half of the patients with metastatic carcinoma, the site of the primary tumour was ascertained. These findings lead us to conclude that, in patients with a suspected skeletal metastasis, the search for the primary tumour should start with FNAB for cytological diagnosis and not with radiological or other investigations which mostly prove to be non-contributory.

To our knowledge our consecutive series of patients with bone lesions is the largest and third largest study of the value of FNAB in the diagnosis of myeloma or lymphoma and metastatic carcinoma, respectively. ${ }^{5,7}$ It may not, however, be considered representative for all patients who present with skeletal lesions since our department is a tertiary referral centre and many patients are referred after initial investigations have failed to give a diagnosis. This may explain why there were few patients with metastases from breast or prostatic carcinoma since these tumours are often diagnosed by clinical examination plus mammography and the level of prostate-specific antigen, respectively. The most common origin of the metastatic carcinomas proved to be the lung (33\%) and kidney (33\%). This predominance may depend on the inaccessibility of these organs to physical examination, the large size to which tumours in these sites can grow before there are symptoms and their propensity to metastasise early to the skeleton. We had more renal carcinomas than in other reported series and fewer gastrointestinal neoplasms.

The risks of open biopsy include infection, bleeding (especially in metastases from renal carcinoma), weakening of the bone leading to pathological fracture, contamination of surrounding soft tissues, and discomfort associated with

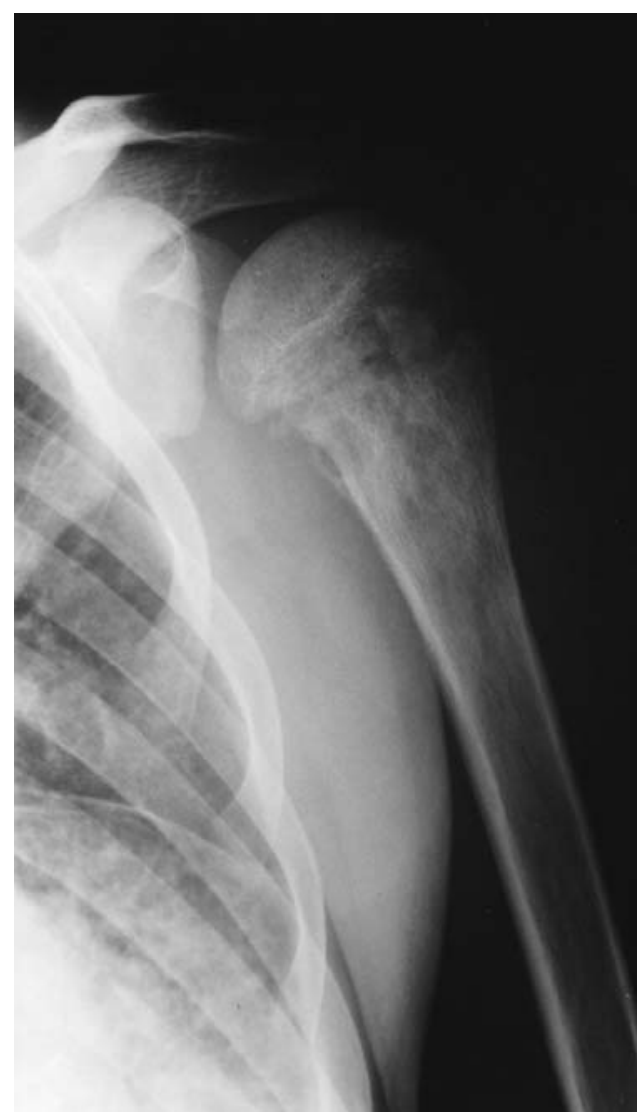

Fig. 2

A 15-year-old boy injured his right shoulder in an accident on a moped. The initial radiographs showed a small lytic lesion considered to be due to the injury. He was treated with a sling and recovered fully. The pain in the shoulder recurred and radiographs 2.5 months later revealed an enlargement of the lytic lesion. MRI showed a soft-tissue component and the patient was referred to us for FNAB. After treatment in the paediatric oncology department, he is alive and well two years after diagnosis of a high-grade lymphoma.

surgery. These disadvantages can be avoided if FNAB is the initial investigation. Furthermore, Bommer et al ${ }^{5}$ have shown that starting the investigation with FNAB is costeffective. The cost of an open biopsy in the USA has been estimated to be \$US5300 as compared with \$US1600 for FNAB, if both are carried out as outpatient procedures. FNAB can be readily done in any skeletal location and it is not necessary to search for a more easily accessible lesion by bone scintigraphy.

The result of cytological diagnosis based on FNAB is strongly related to the experience of the cytologist. The Orthopaedic Oncology Service at the Karolinska Hospital has a long tradition of FNAB, which has been the preferred method of biopsy since the mid 1980s. Using cytomorphology alone, the accuracy was high in suggesting the correct primary tumour in metastatic carcinoma of the kidney (22 of 26), prostate (5 of 6), and breast ( 2 of 2), while guidance to the primary tumour was only possible in 13 of 26 cases of lung cancer and rarely in metastatic carcinoma of other 

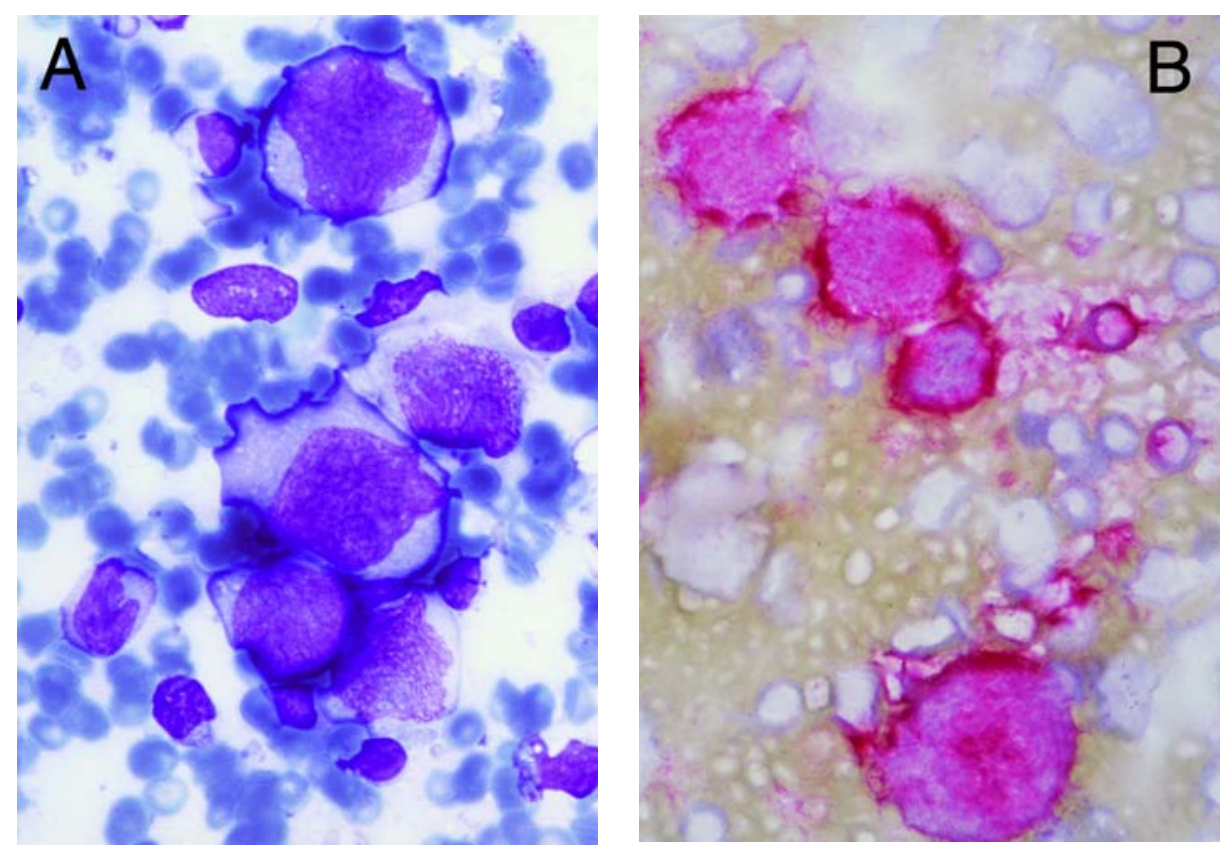

FNAB smear from the proximal humerus of the patient in Figure 2 showing A) immature lymphoid cells from a highgrade malignant lymphoma in contrast to small mature benign lymphocytes (MGG $\times 140$ ) and B) immunostaining on a cytospin preparation showing large immature cells stained with B-cell marker CD20 (alkaline phosphatase $\times 140$ ).

Fig. 3
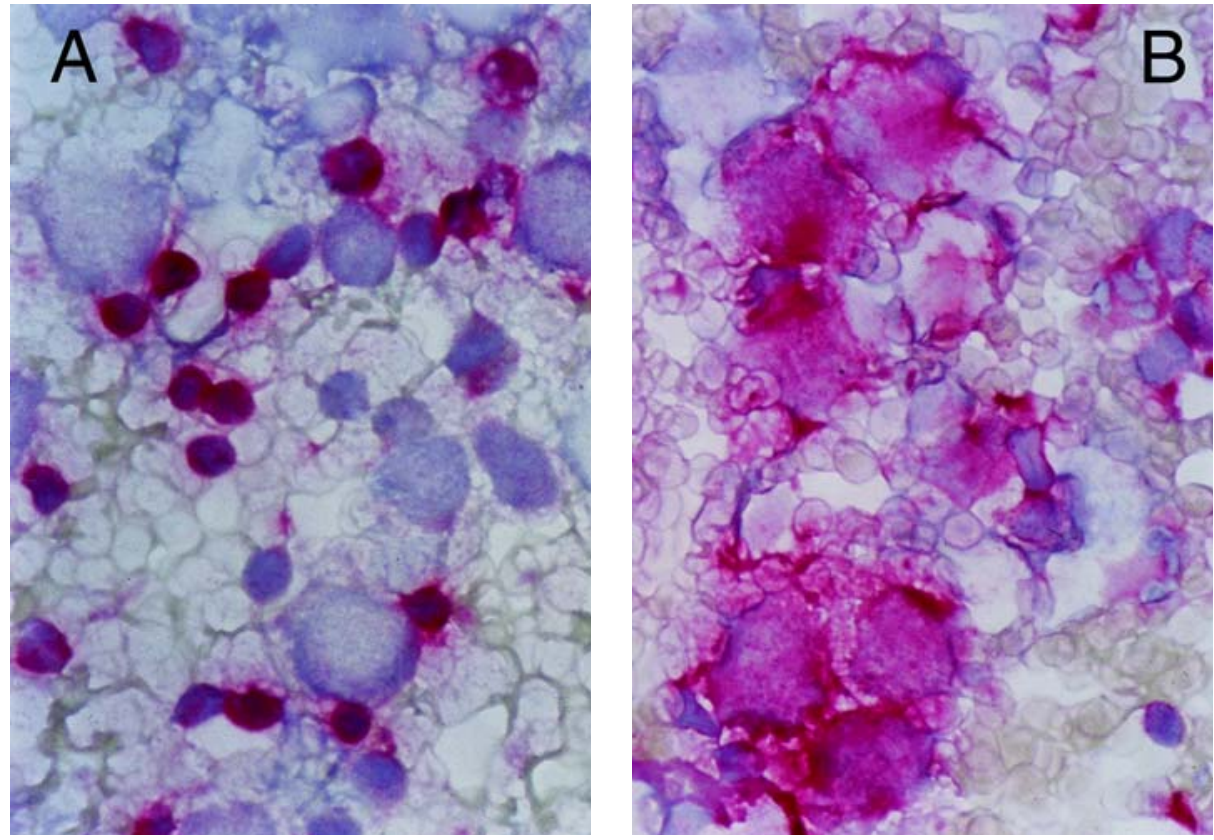

The same case as Figure 3 showing A) immunostaining on cytospin preparation for T-cells and B) large immature cells showing kappa light restriction (alkaline phosphatase $\times 140$ )

Fig. 4

primary tumours. According to several reports the diagnostic accuracy will be considerably increased by applying cytogenetic and immunohistochemical analyses to the cytological material. ${ }^{10-12}$ In 11 cases the primary tumour remained unknown, which compares favourably with the proportion reported in other studies based on surgical biopsies. $1,8,9$

In eight of the 110 patients, the FNAB diagnosis was inconclusive, but did not lead to inappropriate or delayed treatment for any of the patients. As with open biopsy, the cytological assessment must correlate with the clinical and radiological findings. In three cases, no tumour cells were found in the aspirates and therefore other diagnostic methods were employed. In two cases smears showed only tumour necrosis and the surgical biopsies resulted in histological diagnoses. It should be pointed out, however, that in such circumstances a repeat FNAB may also be considered, since it is quicker and less traumatic than an open biopsy. In one case, the aspirates contained necrotic material which was interpreted as most likely representing a carcinoma. 


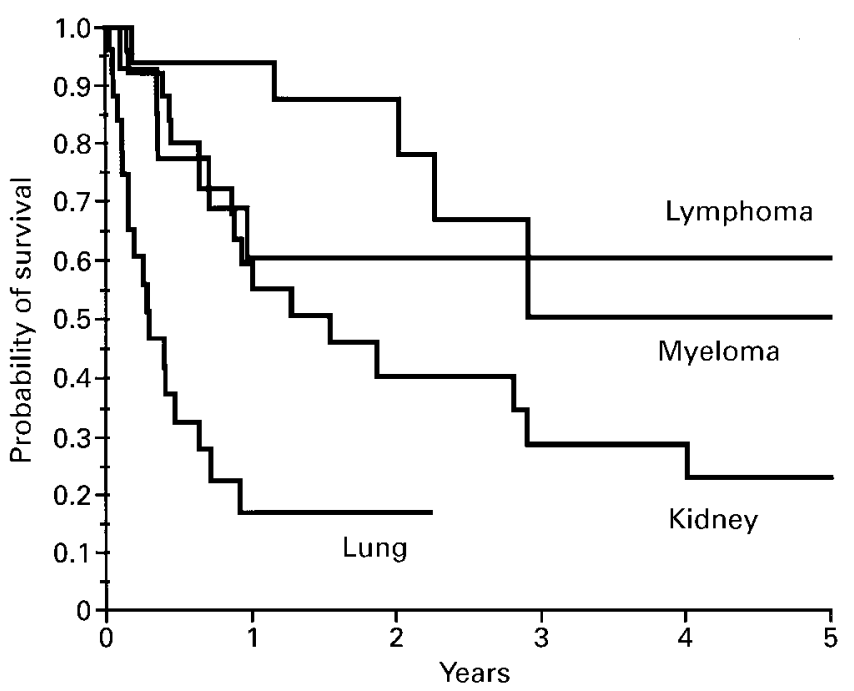

Fig. 5

Rate of survival after diagnosis of the 110 patients.

This underlines the cytological dogma that necrotic material should only be given a diagnosis of tumour necrosis (non-specific) and that no subtyping should be performed. In two cases, the cytology showed tumours which were difficult to subclassify. In both, the primary histology was incorrect compared with the consensus diagnosis. In one patient, the cytological diagnosis correlated with the consensus diagnosis while the primary histological diagnosis was incorrect. It should therefore be concluded that a morphological diagnosis should only be attempted on vital tumour material and that a correct diagnosis is dependent on the experience of the morphologist and not on the type of technique used to obtain tumour tissue. Nowadays, the cytological and histological diagnosis of such cases of undifferentiated malignant tumours is corroborated by immunohistochemistry. We have previously shown that immunocytochemistry allows the correct phenotyping of many malignant tumours. ${ }^{12-15}$ In addition, in the case of metastatic carcinoma, a cytokeratin subtyping will often correctly identify the primary tumour. ${ }^{10}$

The identification of the origin of the skeletal lesion has important therapeutic implications. Skeletal lesions in myeloma or lymphomas rarely need surgical treatment as most instances can be managed by radiotherapy or chemotherapy. Patients with renal carcinoma proved to have a relatively long survival while patients with lung neoplasms had a median survival of only three months after diagnosis. Consequently, we are hesitant to operate on patients with carcinoma of the lung for impending pathological fracture or compression of the spinal cord. Patients with a solitary metastatic lesion and long life expectancy, such as those with renal or thyroid cancer, may be candidates for wide resection and reconstruction in an orthopaedic oncology unit.

FNAB has reduced the frequency of surgical biopsy at our institution from $46 \%$ (1986 to 1990) to $16 \%$ (1997 to 1998) and between 1997 and 1998, the rate of open biopsy was $20 \%$ for primary bone tumours and $2 \%$ for metastatic lesions.

This study was supported by a grant from The Cancer Society in Stockholm, The King Gustav V Jubilee Fund and Dagmar Ferbs fund.

Although none of the authors have received or will receive benefits for personal or professional use from a commercial party related directly or indirectly to the subject of this article, benefits have been or will be received but are directed solely to a research fund, foundation, educational institution, or other non-profit institution with which one or more of the authors is associated.

\section{References}

1. Rougraff BT, Kneisl JS, Simon MA. Skeletal metastases of unknown origin: a prospective study of a diagnostic strategy. J Bone Joint Surg [Am] 1993;75-A:1276-81.

2. Alcalay M, Azais I, Brigeon B, et al. Strategy for identifying primary malignancies with inaugural bone metastases. Rev Rhum Engl Ed 1995;62:632-42.

3. Jacobsen S, Stephensen SL, Paaske BP, Lie PG, Lausten GS. Skeletal metastases of unknown origin: a retrospective analysis of 29 cases. Acta Orthop Belg 1997;63:15-22.

4. Kreicbergs A, Bauer HCF, Brosjö O, et al. Cytological diagnosis of bone tumours. J Bone Joint Surg [Br] 1996;78-B:258-63.

5. Bommer KK, Ramzy I, Mody D. Fine-needle aspiration biopsy in the diagnosis and management of bone lesions: a study of 450 cases. Cancer 1997;81:148-56.

6. Ruhs SA, El-Khoury GY, Chrischilles EA. A cost minimization approach to the diagnosis of skeletal neoplasms. Skeletal Radiol 1996;25:449-54.

7. Åkerman M, Domanski HA. Fine needle aspiration (FNA) of bone tumours: with special emphasis on definitive treatment of primary malignant bone tumours based on FNA. Curr Diagn Pathol 1998;5:82-92.

8. Didolkar MS, Fanous N, Elias EG, Moore RH. Metastatic carcinomas from occult primary tumours: a study of 254 patients. Ann Surg 1977; 186:625-30.

9. Nottebaert M, Exner GU, Hochstetter AR, Schreiber A. Metastatic bone disease from occult carcinoma: a profile. Int Orthop 1989;13:119-23.

10. Wang NP, Zarbo RJ, Bacchi CE, Gown AM. Coordinate expression of cytokeratins 7 and 20 defines unique subsets of carcinomas. Appl Immunohistochem 1995;3:99-107.

11. Miettinen M. Keratin 20: immunohistochemical marker for gastrointestinal, urothelial, and merkel cell carcinomas. Mod Pathol 1995;8:384-8.

12. Skoog L, Tani E. The role of fine needle aspiration cytology in the diagnosis of non Hodgkin's lymphoma. Diagn Oncol 1991:12-19.

13. Tani E, Borregon A, Humla S, Skoog L. Estrogen receptors in fineneedle aspirates from metastatic lesions of gynecologic tumours. Gynaecol Oncol 1989;32:365-7.

14. Skoog L, Schmitt F, Tani E. Neuroendocrine (Merkel-cell) carcinoma of the skin: immunocytochemical and cytomorphologic analysis of fine-needle aspirates. Diagn Cytopathol 1990;6:53-7.

15. Nasiell K, Tani E, Skoog L. Fine needle aspiration cytology and immunohistochemistry of metastatic melanoma. Cytopathol 1991;2:137-47. 